\title{
Robotic On-Orbit Servicing - DLR's Experience and Perspective
}

\author{
DLR (German Aerospace Center) \\ Member of the Helmholtz Association \\ Institute of Robotics and Mechatronics \\ Oberpfaffenhofen, D-82234 Wessling \\ Phone +49 8153 28-2403, Fax +49 8153 28-1134 \\ Email: Klaus.Landzettel@dlr.de
}

K. Landzettel, C. Preusche, A. Albu-Schäffer, D. Reintsema, B. Rebele, G. Hirzinger

\begin{abstract}
The increasing number of launched satellites per year, calls for solutions to keep free operational space for telecommunication systems in geo-synchronized orbit, as well as to avoid the endangering of space systems in LEO (Low-Earth Orbit) and of the public living in the habited parts on Earth. Examples for such dangerous stranded space systems in the past are Skylab and MIR. In the future, the uncontrolled and accidental de-orbiting of other huge satellites is expected, where parts of these will hit the surface of the Earth.
\end{abstract}

A feasible way to handle such problems might be to enforce the operational requirement to use some dedicated residual fuel for a controlled de-orbiting, or in case of GEO (Geostationary Orbit), to lift the satellites at their end of life into the graveyard orbit. Despite these measures, malfunctions of solar generators, control systems or thrusters cannot be avoided. Therefore, on-orbit servicing (OOS) will be a mandatory and challenging topic for space robotics in the near future.

The outcome of national German projects like ROTEX, ESS and GETEX/ETS-VII represent a know-how which can be directly applied for the development of OOS-Robotic systems. Control structures and several possible operational modes are discussed within this paper. The recently started national project ROKVISS already provides the necessary space-qualified hardware as well as the very powerful telepresence operational mode. The paper will concentrate on a description of the ROKVISS mission

The space robotic mission TECSAS (Technology Satellite for Demonstration and Verification of Space Systems), is a very good opportunity to demonstrate and verify satellite capturing, for various degrees of interaction between the robot and the satellite controllers and for various degrees of target cooperation.

The first business case for on-orbit servicing will hopefully become reality within the next few years. DLR's industrial partner ,Orbital Recovery Corp.' has initiated its so-called Orbital Life Extension System (CX-OLEV ${ }^{\mathrm{TM}}$ ). The CXOLEV will operate as an orbital tugboat, supplying the propulsion, navigation and guidance to keep a telecommunications satellite in its dedicated orbital slot for several additional years (station keeping). A short SLES mission description will be given afterwards.
The purpose of ROKVISS, Germans new space robotics technology experiment is to provide robotic hardware components as well as tele-robotic control methods to be applied in the near future for on orbit servicing (OOS). ROVISS was successfully installed outside at the Russian Service Module of the International Space Station (ISS) during an extravehicular space walk at the end of January 2005. Since February 2005 the two-joint manipulator can be operated from ground via a direct radio link. The aim of ROKVISS is the in flight verification of highly integrated modular robotic joints as well as the demonstration of different control modes, reaching from high system autonomy to force feedback teleoperation (telepresence mode). The experiment was already operated for one year in free space and evaluated and qualified the intelligent light weight robotics components under realistic circumstances.

\section{ROKVISS EXPERIMENT DESCRIPTION}

After ROTEX (the first remotely controlled space robot on board of the shuttle COLUMBIA), ROKVISS is the second space robot experiment proposed and realised by DLR's Institute of Robotics and Mechatronics (DLR-RM) in cooperation with the German space companies EADS-ST, Kaiser-Threde, and vHS (von Hörner \& Sulger) with close collaboration of the Russian Federal Space Agency ROSKOSMOS and RKK Energia. While the project was started in 2002, the ROKVISS hardware was mounted outside at the Russian Service Module of the ISS in January 2005. Since February 2005 ROKVISS is operated by DLR-RM, close supported by ZUP, the ISS ground station in Moscow.

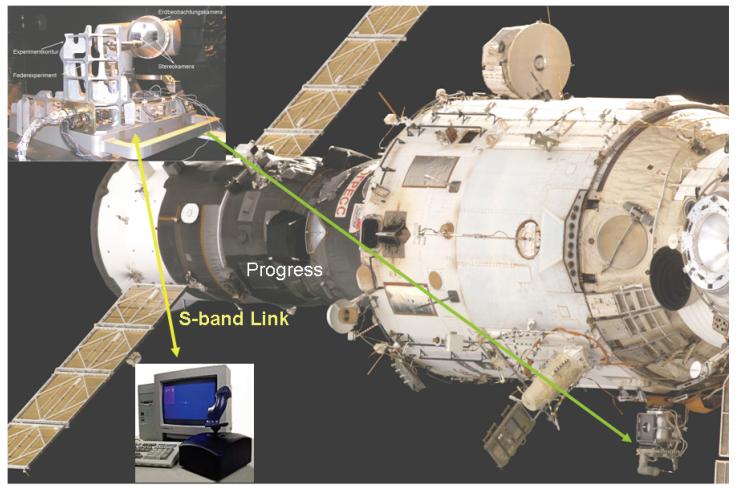

Fig. 1 ROKVISS manipulator with the contour following environment on ISS (ISS-photo by courtesy of NASA) 
The ROKVISS experiment consists of a small robot with two torque-controlled joints (Fig. 2), mounted on an Universal Workplate (UWP), a controller, a stereo camera, an illumination system, an earth observation camera, a power supply, and a mechanical contour device for verifying the robot's functions and performance (Fig. 1). These two robot joints are extensively tested and identified (dynamics, joint parameters) by repetitively performing predefined robot tasks in an automatic mode, or based on direct operator interaction. The automatic mode is necessary due to the fact that communication constraints limit the direct link experiment time to windows of only up to seven minutes length, when the ISS passes over the tracking station German Space Operations Center (GSOC).

The main goals of the ROKVISS [ 2 ] experiment are:

- the verification of DLR's modular light-weight, torque-controlled robotic joints in outer space, under realistic mission conditions, and the identification of their dynamic and friction behaviour over time; The joints are based on DLR's new high energy motor ROBODRIVE and they are identical to those used in DLR's seven joint light weight robot, which for us is the basis for future "robonauts" (Fig. 2 Fig. 17).

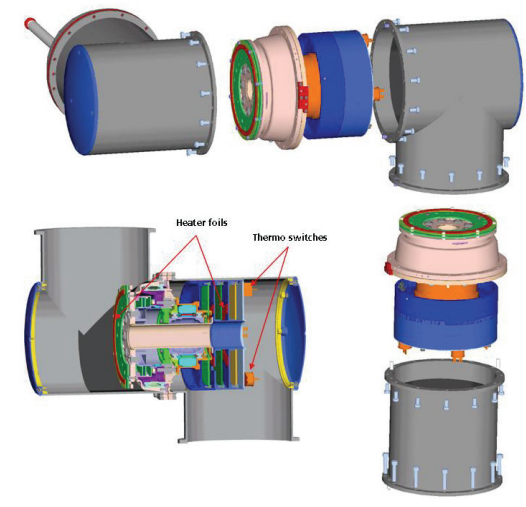

Fig. 2 The two-joint ROKVISS manipulator

- the verification of force-reflecting telemanipulation to show the feasibility of telepresence methods for future satellite servicing tasks [ 3$][5]$ (Fig. 3).

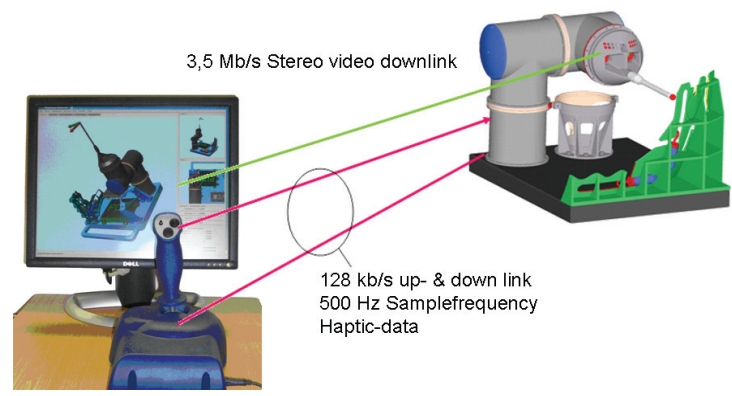

Fig. 3 Telepresence based upon DLR's high-fidelity force reflecting joystick and stereo visual feedback

\section{THE DLR LIGHT WEIGHT JOINTS CONCEPT}

The joint drive requirements in robotics are different from many other applications:

- high torques, not high speed but high dynamics (accelerations),

- frequent motion reversals around the zero position.

Space robotics adds additional requirements as low weight, and low power losses. Thus, an optimised electric motor with respect to the above criteria was developed, using the latest results in concurrent engineering. Finite element technologies were used to model diverse parameter influences on motor performance, such as iron thickness. The result was a motor design yielding a hardly believable $50 \%$ reduction in weight and power loss compared to the best commercially available motors we had used so far (Fig. 4).

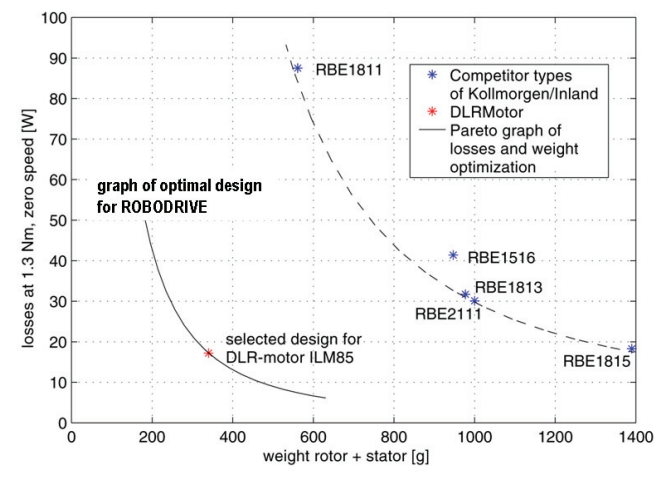

Fig. 4 Comparing ROBODRIVE with the best commercially available motors

\section{QUALIFICATION CONCEPTS}

One basic idea for the ROKVISS project was to get rid of bulky and most expensive radiation-hard components for a space born application in favor of highly integrated circuits used with terrestrial devices. The drawback for using such components is the probability of so called latch-ups due to a CMOS circuit being hit by a heavy ion or proton.

Based on the fact, that neither a hot nor a cold redundant configuration for the joint electronics was intended, a latch-up protected power supply circuit had to be implemented. The task of a latch up protection circuit is to prevent burn out of the device hit and hence to protect it. It is self-evident that the power supply itself must be latch up immune and able to handle latch up situations. Therefore it must be built with radiation tolerant parts in order to guarantee the correct functionality during the whole mission. Meanwhile, within one year of operation we had 1 latchup in joint electronic 2 without any influence on the electronic components. 


\section{OVERALL COMMUNICATION}

In order to keep the round-trip communication time as low as possible, ROKVISS has its own S-band communication system, including an own antenna, pointing to the earth. The overall uplink channel-data rate is $256 \mathrm{kbit} / \mathrm{s}$ whilst the downlink data rate is $4 \mathrm{Mbit} / \mathrm{s}$, including 3,5 Mbit/s videodata.

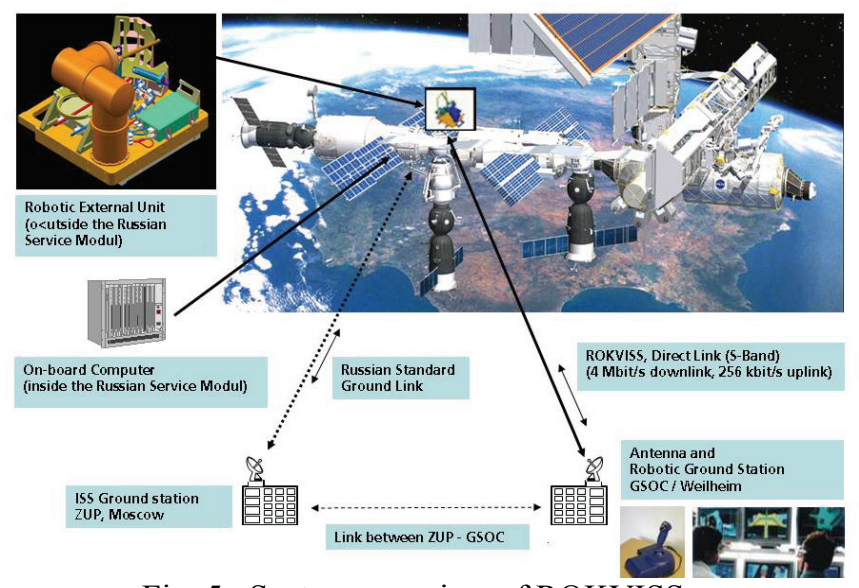

Fig. 5 System overview of ROKVISS

The ROKVISS ground control computers are directly coupled to the transceiver system of DLR's tracking station in Weilheim. The measured round trip times of less than $20 \mathrm{~ms}$ are a very good basis to evaluate the telepresence system behaviour

In order to provide real-time data transmission and to keep the round-trip communication time as low as possible, ROKVISS has access to CUP, a dedicated S-Band communication system with an own antenna, pointing to the earth. Via this S-band radio link the ROKVISS experiments like telepresence, data downloads as well as software and configuration uploads are operated online from ground. The downlink provides a data rate of $4 \mathrm{Mbps}$ for telemetry data like housekeeping data or video images. Depending on the performed operation the downlink data rate sums up approximately by $128 \mathrm{kbps}$ for robot control data and 3,5 Mbps for digital video data, remaining resources are filled by supervisor, miscellaneous data (e.g. housekeeping data or logging messages) and at least the CCSDS protocol overhead.

\section{TELEPRESENCE MODE EXPERIMENTS}

For the telepresence demonstration a stereo camera is mounted on the 2nd joint: The stereo video images, together with the current robot joint and torque values, are fed back in real-time to an operator at the ground station. The operator controls the slave robot at the remote site via a forcefeedback-control device. Force and position commands are generated to drive the robot joints into the desired state. Using high-rate, low latency and jitter-free up- und downlink channels, the operator is "impressively" included in the control loop. A major requirement for this mode is to keep the total data round-trip-delay significantly below 500 millisec- onds. Indeed for the ROKVISS direct over-flight situation we have only $10-20 \mathrm{~ms}$ roundtrip delay, but we will simulate additional time-delays, which occur when a geostationary communication satellite is used.

While large and varying time delay can be compensated by model-based approaches, e.g. using predictive simulation (as was the case in ROTEX), we have a strong signal-based coupling between the ground operator and the space robot in ROKVISS. The force feedback loop is realised with a common position/force control architecture [ 6 ].

To cope with longer and time-varying time-delays, sophisticated bilateral control schemes have to be used. For the ROKVISS experiment an approach using wave-variables has been developed [ 1 ], in which the time-varying delay due to the orbit of the ISS is simulated and compensated. Alternatively a time domain passivity control scheme has been developed. A distributed passivity observer monitors the passivity of the communication channel. In case of activity, which will destabilize the master-slave system, a passivity control acts to maintain stability.

Our evaluation contour provides several experiments to verify our new control schemes under realistic space conditions:

- the contour itself represents a hard surface, which can be contacted with a finger;

- different geometric forms are included for contour following tasks;

- a 2-DoF "Peg-in-Hole" part in the contour realises a 3side-mechanical binding of the touch finger. This represents a typical benchmark for telerobotic applications;

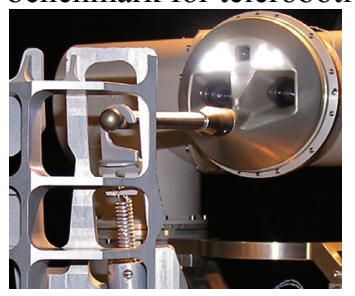

Fig. 6 Spring device for energy storage and friction parameter identification

- mechanical springs simulate an external energy storage, adding energy to the Master-Slave system (Fig. 6);

- A virtual spring is programmed into the robot joints to emulate contour materials of different consistence. The operator has to follow a well-defined virtual path which fades into the video display. The programmed joint impedances are fed back as feedback forces.

Already during the first mission contacts also telepresence experiments as described above have been conducted. The stereo video transmission provides a realistic 3D imagery of the scene, though only as grey image. The presence feeling was improved by the realistic force-feedback provided by the DLR-Joystick. Preliminary results are presented in [ 4 ]. 


\section{AUTOMATIC MODE EXPERIMENTS}

The following automatic mode experiments (independent of direct RF contact) are conducted:

- Predefined trajectories without force contact;

- Predefined trajectories with force contact, i.e., contour tracking or movement against spring load, as described in the telepresence mode section.

- Predefined trajectories with a change from non-contact to contact condition (contact dynamics experiment).

Fig. 7 shows the situation drawing the spring.

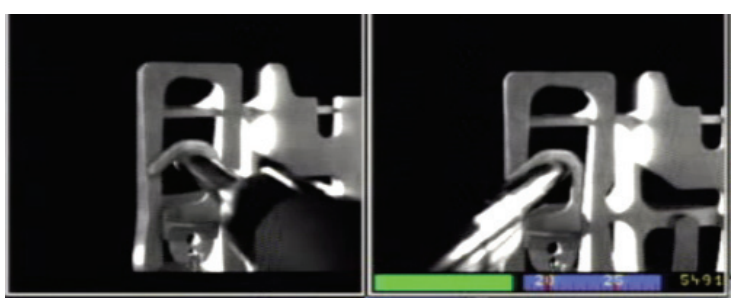

Fig. 7 ROKVISS video images

With the automatic mode experiments, comparable sets of measurement data are generated aiming to analyze the long term behaviour of the robotic joints in free space.

\section{JOINT CONTROLLER STRUCTURE}

The controller structures have initially been developed and verified on the basis of the DLR light-weight robots. In particular a flexible-joint model is assumed. Fast and reliable methods for the identification of the joint model parameters (joint stiffness, damping and friction) were developed, while the rigid body parameters are directly generated from the mechanical CAD programs [ 7$][8]$. This lead to an accurate simulation of the robot dynamics on ground, so that it was possible to develop and test the controller structures in the simulation first.

The basic joint level controller is a joint state feedback controller with compensation of gravity and friction [ 9 ]. The state vector contains the motor position, the joint torques, as well as their derivatives. By the appropriate parameterisation of the feedback gains, the controller structure can be used to implement position, torque or impedance control. The gains of the controller can be computed in every Cartesian cycle, based on the desired joint stiffness and damping, as well as depending on the actual value of the inertia matrix. Hence, this controller structure fulfils the following functionalities:

- It provides active vibration damping of the flexible joint structure;

- It maximises the bandwidth of the joint control for the given instantaneous values of the inertia matrix;

- It implements variable joint stiffness and damping.

Based on this joint control structure, three different strategies for implementing Cartesian compliant motion have been realised (Fig. 8): admittance control, which accesses the joint position interface through the inverse kinematics; impedance control, which is based on the joint torque interface; and Cartesian stiffness control, which accesses the joint impedance controller [ 10 ].

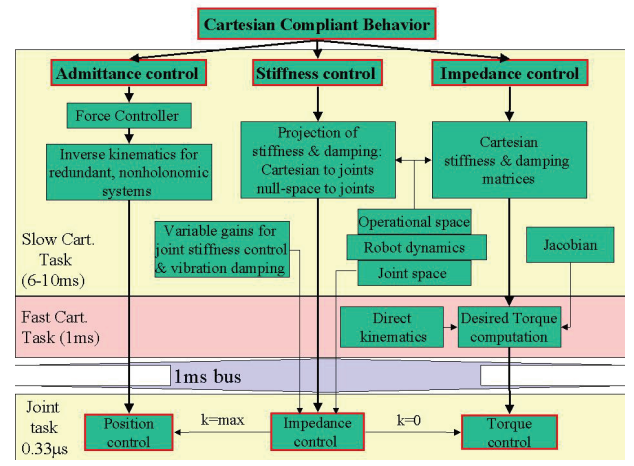

Fig. 8 Controller architecture

The position controller as well as the impedance controller is based on a passivity approach under consideration of the joint flexibilities [ 11 ], [ 12 ]. A physical interpretation of the joint torque feedback loop has been given as the shaping of the motor's kinetic energy, while the implementation of the desired stiffness can be regarded as shaping of potential energy. Therefore, the Cartesian impedance controller can be designed and analyzed within a passivity based framework in the same manner as the joint state feedback controller. This constitutes a new, unified approach for the torque, impedance and position control on both joint and Cartesian level.

An important advantage of these passivity-based controllers is the robustness with respect to uncertainties of the robot or load parameters, as well as to contact-situations with unknown but passive environments.

Despite of the fact that the Cartesian controllers are of limited practical use for a two DoF robot, the entire control structure of the DLR light-weight robots has been implemented also for the ROKVISS system, in order to validate the software for further missions implying robots with six or seven DoF.

\section{JOINT PARAMETER IDENTIFICATION}

Accurate dynamic models were required for both the premission development and testing phase as well as the in-flight operations phase. By using 3D CAD programs for the mechanical design, the problem of determining the parameters of the rigid robot dynamics becomes straightforward, since they can be generated with high accuracy from the design data. This of course requires a detailed modelling of all components, including motors, gears and electronics. The parameters which still have to be identified are the friction parameters, the motor constant and the joint stiffness. The objectives of the technological experiments during the mission are:

- To identify important dynamic non-linear system parameters in micro-gravity environment and validate the underlying multi-body system models,

- To increase modelling fidelity and hence performance quality for future space robotic missions [ 13 ]. 
For the design of the joint controllers (position, torque, and impedance control), recursive least squares methods have been developed, which identify the stiffness and damping, as well as the friction parameters. Starting from the model and the corresponding identification measurements [14 ], a modified time-efficient, on-line version has been tested on ground and is used during the mission.

To identify the different parameter groups we perform dedicated measurements which enable independent identification for each group [ 7 ]. His procedure avoids complex online optimization problems which, in our earlier experiments, always resulted in local minima, very different form the real physical parameters.

\section{Preliminary Joint Friction Identification Results}

For the identification of the motor side friction the following signals are available: the commanded motor current, the measured motor position and hence, by differentiation, the motor velocity, as well as the measured joint torque. The identification procedure determines the motor torque constant $\mathrm{k}_{\mathrm{m}}$, the Coulomb friction $\tau_{C}$, the friction coefficient for the load dependent component $\mu$, and the viscous friction coefficient $b_{1}$. The identification of these parameters can be formulated as a static, linear optimization problem. This means that for a properly chosen trajectory, which independently excites all parameters, a fast and reliable parameter convergence can be obtained. Such a trajectory is given when pulling the springs of the test setup using a saw-tooth trajectory with different constant velocities (Fig. 7). Due to the variable load torque, the reversion of the movement direction, and the coverage of the entire velocity range, all parameters are well excited. A typical identification plot is given in (Fig. 9) and the numerical results for the friction values (measured on 22.07.05 during an automatic on-orbit experiment) are given in table 1 .

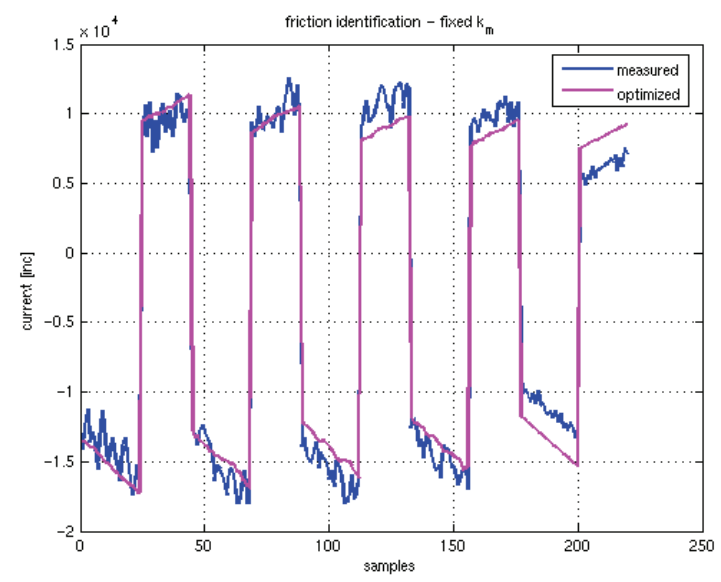

Fig. 9 Commanded current and current estimation after the identification of the friction parameters. The movements are performed with $30.0,20.0,10.0,5.0$, and $1.0 \mathrm{deg} / \mathrm{s}$.

In order to test the reliability of the identified values, the motor constant $\mathrm{km}$ was also identified on a separate motor test-bed and the optimization was performed only for the remaining parameters. Table 1 shows that the results for the two cases are very similar.

Table 1. Friction parameters for joint 1 in space.

\begin{tabular}{|l|c|c|}
\hline & with fixed $\mathrm{k}_{\mathrm{m}}$ & with optimized $\mathrm{k}_{\mathrm{m}}$ \\
\hline $\mathrm{k}_{\mathrm{m}}[\mathrm{Nm} / \mathrm{inc}]$ & 345.62 & 337.39 \\
\hline$\tau_{C}[\mathrm{Nm}]$ & 28.5 & 29.1 \\
\hline$\mu$ & 0.272 & 0.302 \\
\hline $\mathrm{b}_{1}[\mathrm{Nms} / \mathrm{rad}]$ & 12.25 & 12.51 \\
\hline
\end{tabular}

In addition to identifying the parameters of a fixed model, we also try to improve the model itself. For the previously presented triangular trajectory with varying frequency (i.e. velocity amplitude) and undergoing spring load, a nonlinearity of the viscous friction term can be noticed. Fig. 10 compares two further model versions in terms of their degree of complexity and the modelling accuracy on a similar trajectory. Model 1 is a simplified one that accounts only for Coulomb friction and linear viscous damping. Those two damping terms had been identified before in ground experiments to be of dominant influence. The second model, Model 2, is regarded as an improvement of Model 1, and accounts now for more damping terms such as viscous damping of quadratic and cubic velocity dependence, and a term regarding the load dependent effect on Coulomb damping:

$T_{\text {damp }}=\left(\tau_{C}+\mu\left|T_{\text {out }}\right|\right) \operatorname{sign}(v)+b_{1} v+b_{2} v|v|+b_{3} v^{3}$

where $\left|T_{\text {out }}\right|$ is the magnitude of the output torque (e.g. spring load) and $v$ is the angular velocity. The second model fits the measurements considerably better.

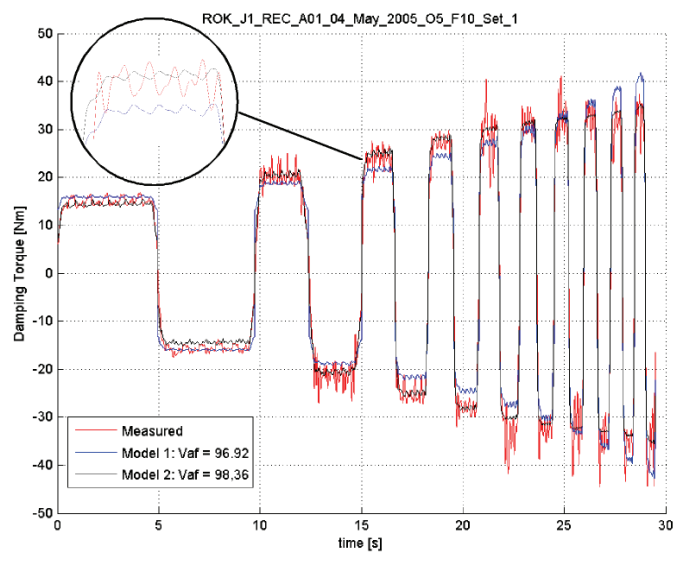

Fig. 10 Two different models for the friction torque are compared. Vaf (= variance accounted for) defines a statistical measure to judge to quality of the identification results and hence the underlying model: The ideal value vaf $=100 \%$ means that we have total agreement between model and measurement. 


\section{Time and Temperature Dependency}

The results of the on-orbit identification show that the total friction for joint 1 in space increased by a factor of about $50 \%$ compared to the friction on ground, taken at $20^{\circ} \mathrm{C}$, under normal atmospheric pressure. However, only o small further continuous change of the parameters can be observed so far over the first year of operation (Fig. 11). This suggests the conclusion that the lubricant changed its properties when exposed to outer space conditions at the beginning of the mission and afterwards reached an operating state with slow parameter variation.

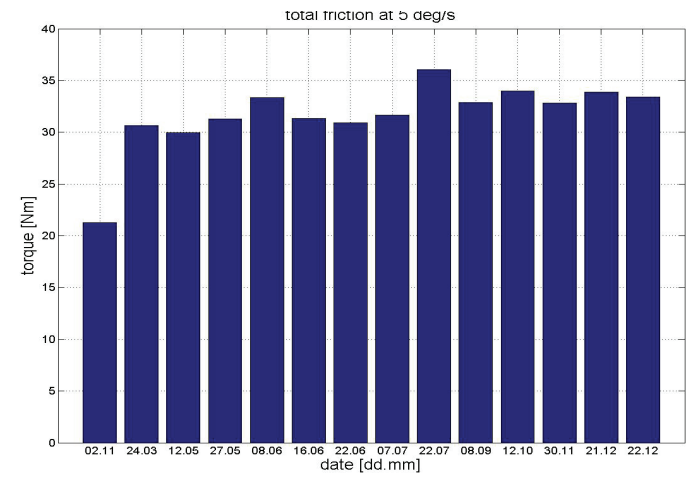

Fig. 11 Time evolution of total friction at a speed of $5 \mathrm{deg} / \mathrm{s}$. The first bar corresponds to the reference measurement on ground.

A heating system is used to regulate the operating temperatures on-orbit between $-20^{\circ} \mathrm{C}$ and $+30^{\circ} \mathrm{C}$. Within this range, a temperature dependency of the parameters is not clearly recognizable. The parameter variation over temperature is thus obviously lower than their variation over time (Fig. 12).

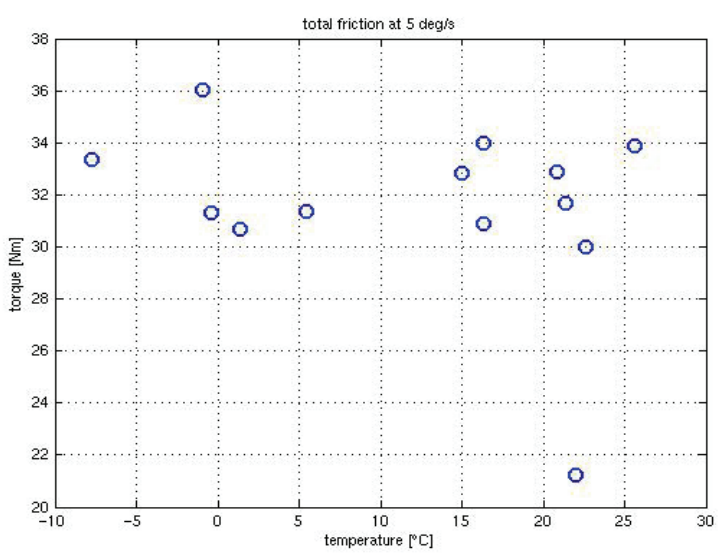

Fig. 12 Total friction at $5 \mathrm{deg} / \mathrm{s}$, plotted over the temperature. The isolated value corresponds to the reference measurement on ground.

\section{Stiffness Identification}

The main sources of elasticity in the joints are the flex splines of the harmonic drives and the torque sensors. The elasticity is identified by contacting a rigid surface with the tip of the robot and commanding a slowly changing force to the joints.
Since the torque is measured after the gear-box, the stiffness can be easily optimized with the available torque and position signal.

A typical identification result can be seen in Fig. 13. The stiffness for both joints has values around $5000 \mathrm{Nn} / \mathrm{rad}$. As expected, the first measurements revealed no significant differences between the stiffness values on ground and in space.

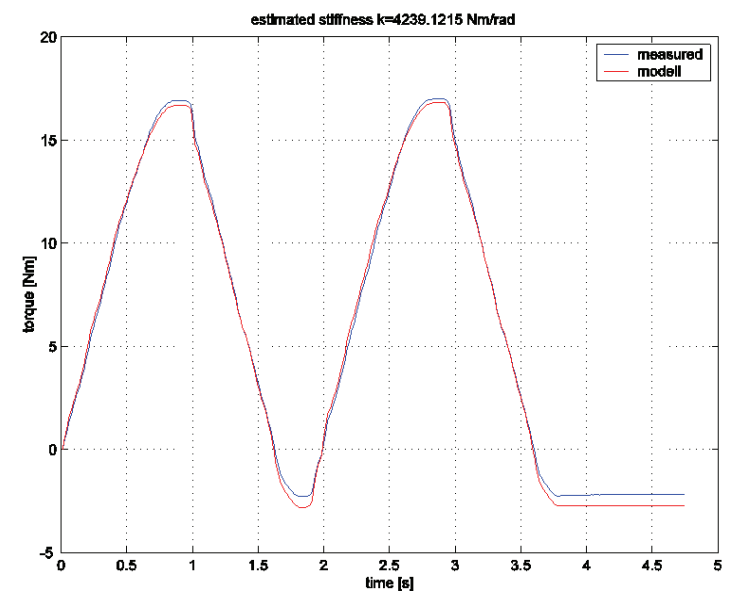

Fig. 13 Result of stiffness optimization.

\section{CONTACT DYNAMICS EXPERIMENTS}

The ROKVISS facility is also used to study how the space environment affects the behaviour of bodies interacting together in a contact situation. Compared with joint dynamics changes, strong dependency of temperature variations and space radiation upon surface material properties is far more expected for the impact and contact cases. In impact and contact dynamics, the proper knowledge of both the energy dissipation and the tangential forces (damping, static and dynamic and friction of Coulomb type) is of our primary interest. These experiments are conducted in cooperation with CSA, the $\mathrm{Ca}$ nadian Space Agency. Specifically, the energy dissipation occurring during intermittent impact events (in normal and oblique impact) is measured, as well as the frictional forces acting between two bodies while they are moving w.r.t. each other in a lasting contact situation (Fig. 14).

The stylus is moved back and forth along a straight edge of the contour in a sinusoidal manner (position control), while the contact normal force is kept constant. The contact forces are recorded for various sinusoidal motion frequencies and normal force amplitudes. The experiment studies the friction behaviour over time of aluminum-aluminum contact as well as the contact between the aluminum tip of the pointer and a lubricated section of the contour. The contact dynamics experiments are performed on an ordinary contour section piece with the nominal surface finish, and on a section of the contour on which a dry-film lubricant has been applied. 


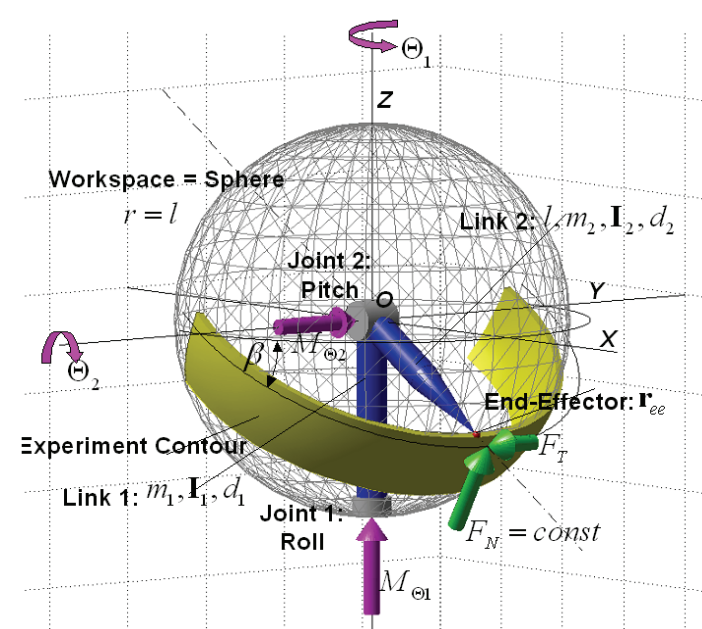

Fig. 14 ROKVISS manipulator: contact case, stylus moving on a given trajectory. This figure is part of an animation: the

length of the arrows is varying according with time. The arrows indicate the torques of the

two joints exerted during motion and the reaction force at the contact surface.

\section{OUTLOOK}

Besides the ROKVISS experiment another space robotics project is going on. The TECSAS (TEChnology SAtellite for demonstration and verification of Space systems) project aims at the in-orbit qualification of the key robotics elements (both hardware and software) for advanced space maintenance and servicing system, especially w.r.t. docking and robot-based capturing procedures.

The Russian Multi-Purposes Orbital Boost Platform as the base module for the mission provides the insertion into initial parking orbit as well as rendezvous and docking maneuvers. For docking and capturing operations we want to use our own robotics means (manipulator based on ROKVISS joints, controller, etc.) as well as MARCO as the ground control environment.

The entire mission will be performed utilizing following steps: far rendezvous, close approach, inspection fly around, capture, stabilization of the compound, compound flight maneuver, manipulation of the target, active ground control via telepresence, passive ground control during autonomous operations (monitoring), and controlled de-orbiting of the compound.

For the capturing of the tumbling target satellite, the control modes will be applied as developed in the ESS study and verified during the ETS-VII mission. After capturing, for compound stabilization, the manipulator can be used as an active damping system: since the platform, including the robot system, and the captured target system build one compound system, the dynamic behavior of the complete system can be influenced by moving the robot. Also for de-orbiting maneuvers the manipulator can be used as a support system: it can be considered as a passive link building the mechanical interface between the chaser and the target system. The geometry of the compound system can be influenced by the robot's attitude. Additionally, the robot can be considered as an active link, thus controlling the thrusters vector pointing to the common center of mass.

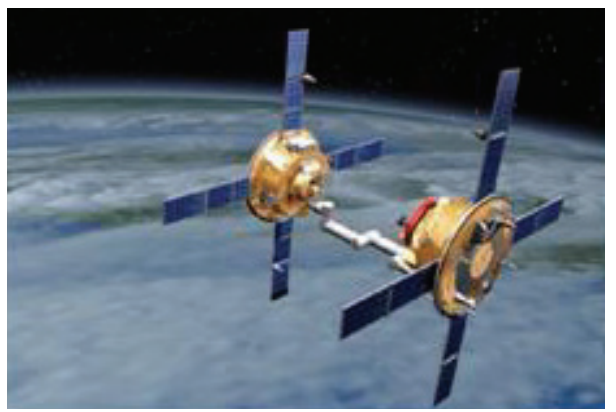

Fig. 15 TECSAS - The freeflying Technologie Satellite for Demonstration and Verification of Space Systems

In contrast to the TECSAS experiment, where the priority is on technological aspects of space robot servicing tasks, we participate on the first business case in on-orbit-servicing, By attaching a "tugboat" to a satellite, whose propellant is declining, the lifetime of valuable telecommunication satellites could be prolonged for several years. Telecommunications satellites typically cost at least $\$ 250$ million - and they are designed for an average useful on-orbit life of 10-15 years. Once their on-board propellant load is depleted, the satellites are boosted into a disposal orbit and decommissioned, even though their revenue-generating communications relay payloads continue to function.

Our industrial partner Orbital Recovery has initiated its socalled Orbital Life Extension System (CX-OLEV $\left.{ }^{\text {TM }}\right)$ which will significantly prolong the operating lifetimes of these valuable telecommunications satellites. The CX-OLEV will operate as an orbital tugboat, supplying the propulsion, navigation and guidance to keep a telecommunications satellite in its proper orbital slot for many additional years. Another application of the CX-OLEV could be the rescue of a spacecraft that have been placed in a wrong orbit by their launch vehicles, or which have become stranded in an incorrect orbital location during positioning maneuvers. The system is designed to easily mate with all telecommunications satellites now in space or on the drawing boards.

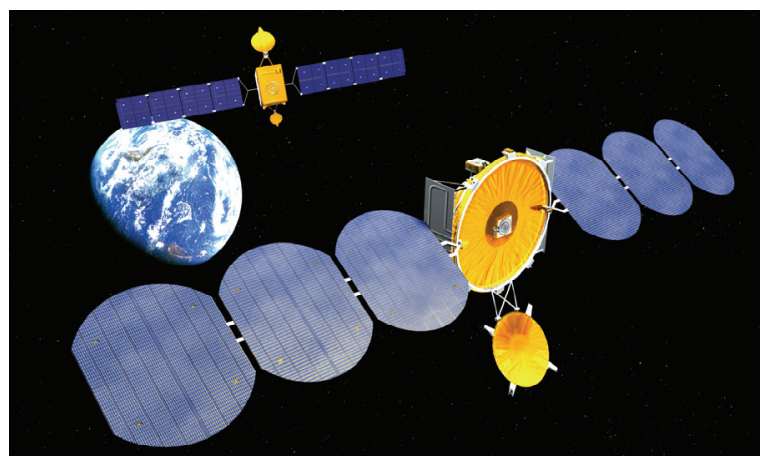

Fig. 16 SLES approaching a telecommunication satellite (Picture by courtesy of Orbital Recovery Corp.) 
It will rendezvous with the telecommunications satellite and link up using our proprietary docking device that connects to the telecommunication satellite's apogee kick motor, as we have proposed within the ESS technology study. Apogee kick motors are used by nearly every telecommunications satellite for orbital boost and they provide a strong, easily accessible interface point for the CX-OLEV.

The already started TECSAS project is the next important step on the roadmap: From our operational terrestrial lightweight arm/hand system (Fig. 17) via ROKVISS and TECSAS towards humanoid free-flying or planetary rover based OOS systems (Fig. 17).
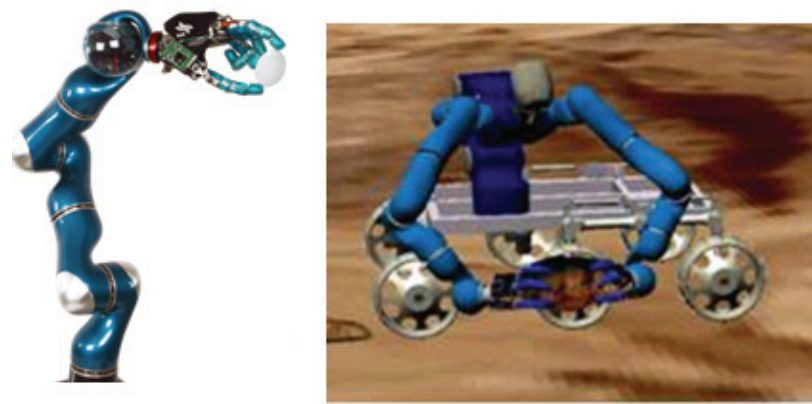

Fig. 17 DLR's operational light-weight arm/hand system (left) and a humanoid planetary rover concept based on DLR's light-weight arm/hand system (right)

\section{REFERENCES}

[ 1 ] Artigas, J., Preusche, C., Hirzinger, G.: Wave Variables based Bilateral Control with a Time Delay Model for Space Robot Applications. Robotik 2004, München, 17.-18.06.2004, VDI, VDI-Bericht 1841, S. 101$108,(2004)$

[ 2 ] Landzettel, K., Brunner, B., Lampariello, R., Preusche, C., Reintsema, G., et al.: System Prerequisites and Operational Modes for On Orbit Servicing. Proc. ISTS International Symposium on Space Technology and Science, Miyazaki, Japan, May 30-June 6, 2004, Proceedings, (2004)

[ 3 ] Preusche, C., Reintsema, D., Landzettel, K., Fischer, M., Hirzinger, G.: DLR on the way towards Telepresent On-Orbit Servicing. Proc. Mechatronics \& Robotics 2004, (2004)

[ 4 ] Preusche, C., Reintsema, D., Landzettel, K., Hirzinger, G.: Robotic Component Verification on ISS (ROKVISS) - Preliminary Results for Telepresence. Proc. of the Int. Conference on Intelligent Robots and Systems, Peking, China, Oct. 2006, (2006)

[ 5 ] Preusche, C., Reintsema, D., Landzettel, K., Hirzinger, G.: ROKVISS - towards Telepresence Control in Advanced Space Missions. Proc. 3rd. International Conference on Humanoid Robots (Humanoids 2003), Munich and Karlsruhe, Oct. 2003, (2003)

[ 6 ] Ortmaier, T.: Telepresence Issues in Minimally Invasive Surgery. Methodology and Tools in Telemanipulation Systems via Internet, E-Book, 32 Pages + DVD-ROM, (2004)

[ 7 ] Albu-Schäffer, A.: Regelung von Robotern mit elastischen Gelenken am Beispiel der DLR-Leichtbauarme. Doctoral thesis, TU München, (2002)

[ 8 ] Borst, C., Fischer, M., Hirzinger, G.: Grasp Planning: How to Choose a Suitable Task Wrench Space. Proc. IEEE International Conference on Robotics and Automation ICRA, New Orleans, USA, April 26 - May 1, pp.319-325, (2004)

[ 9 ] Albu-Schäffer, A., Hirzinger, G.: A globally stable state feedback controller for flexible joint robots. Advanced Robotics, Special Issue, Vol. 15, No. 8, (2001), pp.799-814

[10 ] Albu-Schäffer, A., Hirzinger, G.: Cartesian Impedance Control Techniques for Torque Controlled Light Weight Robots. Proc. IEEE International Conference on Robotics and Automation, Washington D.C, USA, pp.657-663, (2002)

[ 11 ] Albu-Schäffer, A., Ott, C., Hirzinger, G.: A Passivity Based Cartesian Impedance Controller for Flexible Joint Robots - Part II: Full State Feedback, Impedance Design and Experiments. Proc. IEEE International Conference on Robotics and Automation ICRA, New Orleans, USA, April 26 - May 1, pp.2666-2672, (2004)

[ 12 ] Ott, C., Albu-Schäffer, A., Kugi, A., Stramigioli, S., Hirzinger, G.: A Passivity Based Cartesian Impedance Controller for Flexible Joint Robots Part I: Torque Feedback and Gravity Compensation. Proc. IEEE International Conference on Robotics and Automation ICRA, New Orleans, USA, April 26 - May 1, pp.2659-2665, (2004)

[ 13 ] Schäfer, B., Landzettel, K., Albu-Schäffer, A., Hirzinger, G.: ROKVISS: Orbital Testbed for Tele-Presence Experiments, Novel Robotic Components and Dynamics Models Verification. Proc. 8th ESA Workshop on Advanced Space Technologies for Robotics and Automation (ASTRA), Noordwijk, The Netherlands, Nov. 2-4, (2004)

[14] Schäfer, B., Rebele, B., Landzettel, K.: ROKVISS - Space Robotics Dynamics and Control Performance Experiments at the ISS. Proc. ACA2004 IFAC Symposium on Automatic Control in Aerospace, St . Petersburg, Russia, 13-18 June 2004, pp.333-338, (2004) 